\title{
Abductive Knowledge Induction From Raw Data
}

\author{
Wang-Zhou Dai, Stephen Muggleton \\ Department of Computing, Imperial College London, London, UK \\ \{w.dai, s.muggleton\}@imperial.ac.uk
}

\begin{abstract}
For many reasoning-heavy tasks involving raw inputs, it is challenging to design an appropriate endto-end learning pipeline. Neuro-Symbolic Learning, divide the process into sub-symbolic perception and symbolic reasoning, trying to utilise datadriven machine learning and knowledge-driven reasoning simultaneously. However, they suffer from the exponential computational complexity within the interface between these two components, where the sub-symbolic learning model lacks direct supervision, and the symbolic model lacks accurate input facts. Hence, most of them assume the existence of a strong symbolic knowledge base and only learn the perception model while avoiding a crucial problem: where does the knowledge come from? In this paper, we present Abductive Meta-Interpretive Learning $\left(M e t a_{A b d}\right)$ that unites abduction and induction to learn neural networks and induce logic theories jointly from raw data. Experimental results demonstrate that Meta $_{A b d}$ not only outperforms the compared systems in predictive accuracy and data efficiency but also induces logic programs that can be re-used as background knowledge in subsequent learning tasks. To the best of our knowledge, Meta $a_{A b d}$ is the first system that can jointly learn neural networks from scratch and induce recursive first-order logic theories with predicate invention.
\end{abstract}

\section{Introduction}

Despite the success of data-driven end-to-end deep learning in many traditional machine learning tasks, it has been shown that incorporating domain knowledge is still necessary for some complex learning problems [Dhingra et al., 2020; Grover et al., 2019; Trask et al., 2018]. In order to leverage complex domain knowledge that is discrete and relational, end-to-end learning systems need to represent it with a differentiable module that can be embedded in the deep learning context. For example, graph neural networks (GNN) use relational graphs as an external knowledge base [Zhou et al., 2018]; some works even considers more specific domain knowledge such as differentiable primitive predicates and programs [Dong et al., 2019; Gaunt et al., 2017]. However, it is hard to design a unified differentiable module to accurately represent general relational knowledge, which may contain complex inference structures such as recursion [Glasmachers, 2017; Garcez et al., 2019].

Therefore, many researchers propose to break the end-toend learning pipeline apart, and build a hybrid model that consists of smaller modules where each of them only accounts for one specific function [Glasmachers, 2017]. A representative branch in this line of research is Neuro-Symbolic (NeSy) AI [De Raedt et al., 2020; Garcez et al., 2019] aiming to bridge System 1 and System 2 AI [Kahneman, 2011; Bengio, 2017], i.e., neural-network-based machine learning and symbolic-based relational inference.

However, the lack of supervision in the non-differentiable interface between neural and symbolic systems, based on the facts extracted from raw data and their truth values, leads to high computational complexity in learning [Li et al., 2020; Dai et al., 2019]. Consequently, almost all neural-symbolic models assume the existence of a very strong predefined domain knowledge base and could not perform program induction. This limits the expressive power of the hybrid-structured model and sacrifices many benefits of symbolic learning (e.g., predicate invention, learning recursive theories, and re-using learned models as background knowledge).

In this paper, we integrate neural networks with Inductive Logic Programming (ILP) [Muggleton and de Raedt, 1994] to enable first-order logic theory induction from raw data. More specifically, we present Abductive Meta-Interpretive Learning $\left(\right.$ Meta $\left._{A b d}\right)$ which extends the Abductive Learning (ABL) framework [Dai et al., 2019; Zhou, 2019] by combining logical induction and abduction [Flach et al., 2000] with neural networks in Meta-Interpretive Learning (MIL) [Muggleton et al., 2015]. Meta $a_{A b d}$ employs neural networks to extract probabilistic logic facts from raw data, and induces an abductive logic program [Kakas et al., 1992] that can efficiently infer the truth values of the facts to train the neural model.

To the best of our knowledge, Meta $a_{A b d}$ is the first system that can simultaneously (1) train neural models from scratch, (2) learn recursive logic theories and (3) perform predicate invention from domains with sub-symbolic representation. In the experiments we compare $M e t a_{A b d}$ to the compared state-of-the-art end-to-end deep learning models and neurosymbolic methods on two complex learning tasks. The results 
show that, given the same amount of background knowledge, Meta $a_{A b d}$ outperforms the compared models significantly in terms of predictive accuracy and data efficiency, and learns human interpretable models that could be re-used in subsequent learning tasks.

\section{Related Work}

Solving "System 2" problems requires the ability of relational and logical reasoning [Kahneman, 2011; Bengio, 2017]. Due to its complexity, many researchers have tried to embed intricate background knowledge in end-to-end deep learning models. For example, [Trask et al., 2018] propose the differentiable Neural Arithmetic Logic Units (NALU) to model basic arithmetic functions (e.g., addition, multiplication, etc.) in neural cells; [Grover et al., 2019] encode permutation operators with a stochastic matrix and present a differentiable approximation to the sort operation; [Wang et al., 2019] introduce a differentiable SAT solver to enable gradient-based constraint solving. However, most of these specially designed differentiable modules are ad hoc approximations to the original symbolic inference mechanisms.

To exploit the complex background knowledge expressed by formal languages directly, Statistical Relational (StarAI) and Neural Symbolic (NeSy) AI [De Raedt et al., 2020; Garcez et al., 2019] try to use probabilistic inference or other differentiable functions to approximate logical inference [Cohen et al., 2020; Dong et al., 2019; Manhaeve et al., 2018; Donadello et al., 2017]. However, they require a pre-defined symbolic knowledge base and only train the attached neural/probabilistic models due to the highly complex interface between the neural and symbolic modules.

One way to learn symbolic theories is to use Inductive Logic Programming [Muggleton and de Raedt, 1994]. Some early work on combining logical abduction and induction can learn logic theories even when input data is incomplete [Flach et al., 2000]. Recently, OILP was proposed for learning firstorder logic theories from noisy data [Evans and Grefenstette, 2018]. However, ILP-based works are designed for learning in symbolic domains. Otherwise, they need to use a fully trained neural models to make sense of the raw inputs.

Machine apperception [Evans et al., 2021] unifies Answer Set Programming with perception by modelling it with binary neural networks. It can learn recursive logic theories and perform concept (monadic predicate) invention. However, both logic hypotheses and the parameters of neural networks are represented by logical groundings, making the system very hard to optimise. For problems involving noisy inputs like MNIST images, it still requires a fully pre-trained perceptual neural net to extract the symbols like ILP-based methods.

Previous work on Abductive Learning (ABL) [Dai et al., 2019; Dai and Zhou, 2017] also unites sub-symbolic perception and symbolic reasoning through abduction, but they need a pre-defined knowledge base to enable abduction and cannot perform program induction. Our presented Abductive Meta-Interpretive Learning takes a step further, which not only learns a perception model that can make sense of raw data, but also learns logic programs and performs predicate invention to understand the underlying relations in the task.

\section{Abductive Meta-Interpretive Learning}

\subsection{Problem Formulation}

A typical model bridging sub-symbolic and symbolic learning contains two major parts: a perception model and a reasoning model [Dai et al., 2019]. The perception model maps sub-symbolic inputs $x \in \mathcal{X}$ to some primitive symbols $z \in \mathcal{Z}$, such as digits, objects, ground logical expressions, etc. The reasoning model takes the interpreted $z$ as input and infers the final output $y \in \mathcal{Y}$ according to a symbolic knowledge base $B$. Because the primitive symbols $z$ are uncertain and not observable from both training data and the knowledge base, we have named them as pseudo-labels of $x$.

The perception model is parameterised with $\theta$ and outputs the conditional probability $P_{\theta}(z \mid x)=P(z \mid x, \theta)$; the reasoning model $H \in \mathcal{H}$ is a set of first-order logical clauses such that $B \cup H \cup z \models y$, where " $=$ " means "logically entails". Our target is to learn $\theta$ and $H$ simultaneously from training data $D=\left\{\left\langle x_{i}, y_{i}\right\rangle\right\}_{i=1}^{n}$. For example, if we have one example with $x=[1,2, \boldsymbol{3}$ and $y=6$, given background knowledge about adding two numbers, the hybrid model should learn a perception model that recognises $z=[1,2,3]$ and induce a program to add each number in $z$ recursively.

Assuming that $D$ is an i.i.d. sample from the underlying distribution of $(x, y)$, our objective can be represented as

$$
\left(H^{*}, \theta^{*}\right)=\underset{H, \theta}{\arg \max } \prod_{\langle x, y\rangle \in D} \sum_{z \in \mathcal{Z}} P(y, z \mid B, x, H, \theta),
$$

where pseudo-label $z$ is a hidden variable. Theoretically, this problem can be solved by Expectation Maximisation (EM) algorithm. However, the symbolic hypothesis $H-$ a first-order logic theory-is difficult to be optimised together with the parameter $\theta$, which has a continuous hypothesis space.

We propose to solve this problem by treating $H$ like $z$ as an extra hidden variable, which gives us:

$$
\theta^{*}=\underset{\theta}{\arg \max } \prod_{\langle x, y\rangle \in D} \sum_{H \in \mathcal{H}} \sum_{z \in \mathcal{Z}} P(y, H, z \mid B, x, \theta) .
$$

Now, the learning problem can be split into two EM steps: (1) Expectation: obtaining the expected value of $H$ and $z$ by sampling them in their discrete hypothesis space from $(H, z) \sim P(H, z \mid B, x, y, \theta)$; (2) Maximisation: estimating $\theta$ by maximising the likelihood of training data with numerical optimisation approaches such as gradient descent.

Challenges. The main challenge is to estimate the expectation of the hidden variables $H \cup z$, i.e., we need to search for the most probable $H$ and $z$ given the $\theta$ learned in the previous iteration. This is not trivial. Even when $B$ is sound and complete, estimating the truth-values of hidden variable $z$ results in a search space growing exponentially with the number of training examples, which is verified in our experiments with DeepProblog [Manhaeve et al., 2018] in section 4.1.

Furthermore, $H$ and $z$ are entangled, which makes the tasks even harder. For example, given $x=[\mathbf{1}, \mathbf{2}, \mathbf{3}]$ and $y=6$, when the perception model correctly recognises $z=[1,2,3]$, we have at least two choices for $H$ : cumulative sum or cumulative product. When the perception model is under-trained and outputs $z=[2,2,3]$, then $H$ could be a program that only multiplies the last two digits. 
Example $(\langle x, y\rangle)$ :

$f([1,3,5], 15)$.

Abducible Primitives $(B)$ :

$\operatorname{add}([A, B \mid T],[C \mid T]):-C \#=A+B$.

$\operatorname{mult}([A, B \mid T],[C \mid T]):-C \#=A * B$.

eq $\left(\left[\mathrm{A} \mid \mathrm{C}_{-}\right], \mathrm{B}\right):-\mathrm{A} \#=\mathrm{B}$.

$\operatorname{head}\left(\left[\mathrm{HI} \_\right], \mathrm{H}\right)$.

$\operatorname{tail}\left(\left[\left[_{-} \mid \mathrm{T}\right], \mathrm{T}\right)\right.$.

Neural Probabilistic facts $\left(p_{\theta}(z \mid x)\right)$ :

$\mathrm{nn}(\boldsymbol{1}=0,0.02) . \mathrm{nn}(\boldsymbol{1}=1,0.39)$.

‥

$\mathrm{nn}(3=0,0.09) \cdot \mathrm{nn}(\mathbf{3}=1,0.02)$.

$\cdots$

$\mathrm{nn}(\mathbf{5}=0,0.07) \cdot \mathrm{nn}(\mathbf{5}=1,0.00)$.

...

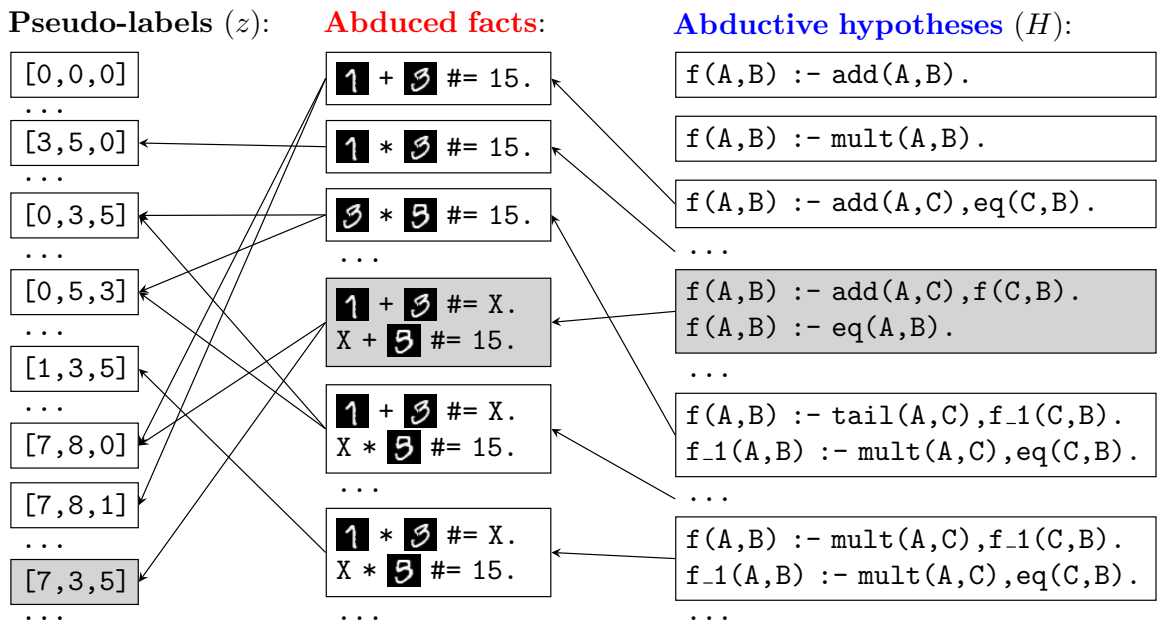

Figure 1: Example of $M e t a_{A b d}$ 's abduction-induction learning. Given training examples, background knowledge of abducible primitives and probabilistic facts generated by a perceptual neural net, Met $a_{A b d}$ learns an abductive logic program $H$ and abduces relational facts as constraints (implemented with the CLP(Z) predicate "\#="1) over the input images; it then uses them to efficiently prune the search space of the most probable pseudo-labels $z$ (in grey blocks) for training the neural network.

\subsection{Probabilistic Abduction-Induction Reasoning}

Inspired by early works in abductive logic programming [Flach et al., 2000], we propose to solve the challenges above by combining logical induction and abduction. The induction learns an abductive logic theory $H$ based on $P_{\theta}(z \mid x)$; the abduction made by $H$ reduces the search space of $z$.

Abductive reasoning, or abduction refers to the process of selectively inferring specific grounded facts and hypotheses that give the best explanation to observations based on background knowledge of a deductive theory.

Definition 3.1 (Abducible primitive) An abducible primitive is a predicate that defines the explanatory grounding facts in abductive reasoning.

Definition 3.2 (Abductive hypothesis) An abductive hypothesis is a set of first-order logic clauses whose body contains literals of abductive primitives.

Following is an example of using abductive hypothesis and abducible primitive in problem-solving:

Example 1 Observing raw inputs $x=[\boldsymbol{2}, \mathbf{3}, \mathbf{1}]$ and a symbolic output $y=6$, we could formulate an abductive hypothesis $H$ that is a recursive cumulative sum function, whose abductive primitives are "+" and "=". Hence, $H$ will abduce a set of explanatory ground facts $\{\mathbf{2}+\mathbf{3}=\mathrm{Z}, \mathrm{Z}+\mathbf{1}=6\}$. Based on these facts, we could infer that none of the digits in $x$ is greater than 6 . Furthermore, if the current perception model assigns very high probabilities to $\mathbf{2}=2$ and $\mathbf{3}=3$, we could easily infer that $\boldsymbol{1}=1$ even when the perception model has relatively low confidence about it.

An illustrative example of combining abduction and induction with probabilities is shown in Fig. 1. Briefly speaking,

\footnotetext{
${ }^{1} \mathrm{CLP}(\mathrm{Z})$ is a constraint logic programming package accessible at https://github.com/triska/clpz. More implementation details in online version: https://arxiv.org/abs/2010.03514.
}

instead of directly sampling pseudo-labels $z$ and $H$ together from the huge hypothesis space, our $M e t a_{A b d}$ induces $a b$ ductive hypothesis $H$ consists of abducible primitives, and then use the abduced facts to prune the search space of $z$. Meanwhile, the perception model outputs the likelihood of pseudo-labels with $p_{\theta}(z \mid x)$ defining a distribution over all possible values of $z$ and helps to find the most probable $H \cup z$.

Formally, we re-write the likelihood of each $\langle x, y\rangle$ in Eq. 2:

$$
\begin{aligned}
P(y, H, z \mid B, x, \theta) & =P(y, H \mid B, z) P_{\theta}(z \mid x) \\
& =P(y \mid B, H, z) P(H \mid B, z) P_{\theta}(z \mid x) \\
& =P(y \mid B, H, z) P_{\sigma^{*}}(H \mid B) P_{\theta}(z \mid x),
\end{aligned}
$$

where $P_{\sigma^{*}}(H \mid B)$ is the Bayesian prior distribution on firstorder logic hypotheses, which is defined by the transitive closure of stochastic refinements $\sigma^{*}$ given the background knowledge $B$ [Muggleton et al., 2013], where a refinement $\sigma$ is a unit modification (e.g., adding/removing a clause or literal) to a logic theory. The equations hold because: (1) pseudo-label $z$ is conditioned on $x$ and $\theta$ since it is the output of the perception model; (2) $H$ follows the prior distribution so it only depends on $B$; (3) $y \cup H$ is independent from $x$ given $z$ because the relations among $B, H, y$ and $z$ are determined by pure logical inference, where:

$$
P(y \mid B, H, z)= \begin{cases}1, & \text { if } B \cup H \cup z \models y, \\ 0, & \text { otherwise. }\end{cases}
$$

Following Bayes' rule we have $P(H, z \mid B, x, y, \theta) \propto$ $P(y, H, z \mid B, x, \theta)$. Now we can search for the most probable $H \cup z$ in the expectation step according to Eq. 3 as follows:

1. Induce an abductive theory $H \sim P_{\sigma^{*}}(H \mid B)$;

2. Use $H \cup B$ and $y$ to $a b d u c e^{2}$ possible pseudo-labels $z$, which are guaranteed to satisfy $H \cup B \cup z \vdash y$ and exclude the values of $z$ such that $P(y \mid B, H, z)=0$;

\footnotetext{
${ }^{2}$ It can be parallelled, see https://arxiv.org/abs/2010.03514.
} 


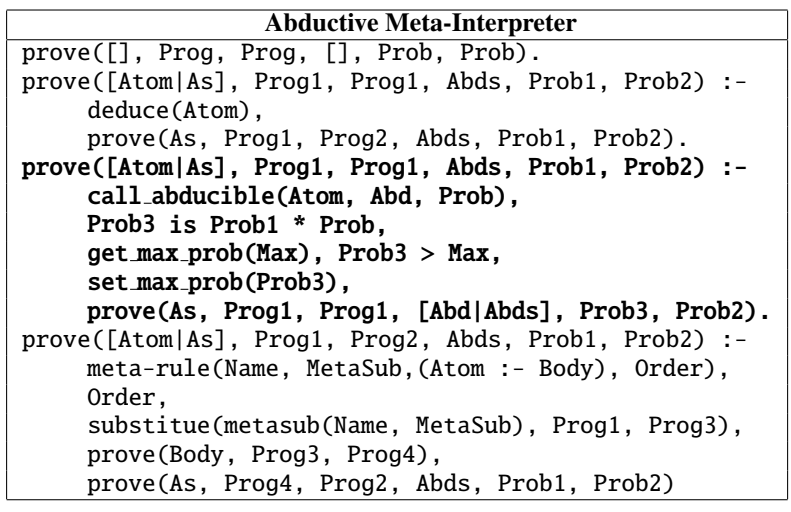

Figure 2: Prolog code for Meta $a_{A b d}$.

3. According to Eq. 3 and 4, score each sampled $H \cup z$ :

$$
\operatorname{score}(H, z)=P_{\sigma^{*}}(H \mid B) P_{\theta}(z \mid x)
$$

4. Return the $H \cup z$ with the highest score.

\subsection{The $M e t a_{A b d}$ Implementation}

Meta-Interpretive Learning [Muggleton et al., 2015] is a form of ILP [Muggleton and de Raedt, 1994]. It learns first-order logic programs with a second-order meta-interpreter, which consists of a definite first-order background knowledge $B$ and meta-rules $M$. $B$ contains the primitive predicates for constructing first-order hypotheses $H ; M$ is second-order clauses with existentially quantified predicate variables and universally quantified first-order variables. In short, MIL attempts to prove the training examples and saves the resulting programs for successful proofs.

Met $a_{A b d}$ extends the general meta-interpreter of MIL by including an abduction procedure (bold fonts in Fig. 2) that can abduce groundings (e.g., specific constraints on pseudolabels $z$ ). As shown in Fig. 2, it recursively proves a series of atomic goals by deduction (deduce/1), abducing explanatory facts (call_abducible/3) or generating a new clause from meta-rule/4.

The last argument of call_abducible/3, Prob = $P_{\theta}(z \mid x)$, describes the distribution of possible worlds collected from the raw inputs. It helps pruning the search space of the abductive hypothesis $H$. During the iterative refinement of $H$, Meta $a_{A b d}$ greedily aborts its current prove/ 6 procedure once it has a lower probability than the best abduction so far (the 8th line in Fig. 2).

After an abductive hypothesis $H$ has been constructed, the search for $z$ will be done by logical abduction. Finally, the score of $H \cup z$ will be calculated by Eq. 5, where $P_{\theta}(z \mid x)$ is the output of the perception model, which in this work is implemented with a neural network $\varphi_{\theta}$ that outputs:

$$
P_{\theta}(z \mid x)=\operatorname{softmax}\left(\varphi_{\theta}(x, z)\right) \text {. }
$$

Meanwhile, we define the prior distribution on $H$ by following [Hocquette and Muggleton, 2018]:

$$
P_{\sigma^{*}}(H \mid B)=\frac{6}{(\pi \cdot c(H))^{2}},
$$

where $C(H)$ is the complexity of $H$, e.g., its size.

\section{Experiments}

This section describes the experiments of learning recursive arithmetic and sorting algorithms from images of handwritten digits ${ }^{3}$, aiming to address the following questions: (1) Can $M e t a_{A b d}$ learn first-order logic programs and train perceptual neural networks jointly? (2) Given the same or less amount of domain knowledge, is hybrid modelling, which directly leverages the background knowledge in symbolic form, better than end-to-end learning?

\subsection{Cumulative Sum and Product From Images}

Materials. We follow the settings in [Trask et al., 2018]. The inputs of the two tasks are sequences of randomly chosen MNIST digits; the numerical outputs are the sum and product of the digits, respectively. The lengths of training sequences are 2-5. To verify if the learned models can extrapolate to longer inputs, the length of test examples ranges from 5 to 100. Note that for cumulative product, the extrapolation examples has maximum length 15 and only contain digits from 1 to 9. The dataset contains 3000 and 1000 examples for training and validation, respectively; the test data of each length has 10,000 examples.

Methods. We compare Meta $a_{A b d}$ with following state-ofthe-art baselines: End-to-end models include RNN, LSTM and LSTMs attached to Neural Accumulators(NAC) and Neural Arithmetic Logic Units (NALU) [Trask et al., 2018]; NeSy system DeepProblog [Manhaeve et al., 2018] ${ }^{4}$.

A convnet processes the input images to the recurrent networks and Problog programs, as [Trask et al., 2018] and [Manhaeve et al., 2018] described; it also serves as the perception model of $M e t a_{A b d}$ to output the probabilistic facts. As shown in Tab. 1, NAC, NALU and Meta $a_{A b d}$ are aware of the same amount of background knowledge for learning both perceptual convnet and recursive arithmetic algorithms jointly, while DeepProblog is provided with the ground-truth program and only trains the perceptual convnet.

Each experiment is carried out five times, and the average of the results are reported. The performance is measured by classification accuracy (Acc.) on length-one inputs, mean average error (MAE) in sum tasks, and mean average error on logarithm (log MAE) of the outputs in product tasks whose error grows exponentially with sequence length.

Results. Our experimental results are shown in Tab. 2; the learned first-order logic theories are shown in Fig. 3a. The end-to-end models that do not exploit any background knowledge (LSTM and RNN) perform worst. NALU and NAC is slightly better because they include neural cells with arithmetic modules, but the end-to-end learning pipeline based on embeddings results in low sample-efficiency. DeepProblog does not finish the training on the cumulative sum task and the test on cumulative product task within 72 hours because the recursive programs result in a huge groundings space for its maximum a posteriori (MAP) estimation.

\footnotetext{
${ }^{3}$ Code \& data: https://github.com/AbductiveLearning/Meta_Abd

${ }^{4}$ We use NAC/NALU at https://github.com/kevinzakka/NALUpytorch; DeepProblog at https://bitbucket.org/problog/deepproblog
} 


\begin{tabular}{|c|c|c|c|}
\hline Domain Knowledge & End-to-end Models & Neuro-Symbolic Models & MetaAbd \\
\hline Recurrence & LSTM \& RNN & Problog's list operations & Prolog's list operations \\
\hline Arithmetic functions & NAC\& NALU [Trask et al., 2018] & Full program of accumulative sum/product & Predicates add, mult and eq \\
\hline Sequence \& Odering & Permutation matrix $P_{\text {sort }}$ [Grover et al., 2019] & Predicates ">", "=" and "<" [Dong et al., 2019] & Prolog's permutation \\
\hline Sorting & sort operator [Grover et al., 2019] & $\operatorname{swap}(i, j)$ operator [Dong et al., 2019] & Predicate s (learned from sub-task) \\
\hline
\end{tabular}

Table 1: Domain knowledge used by the compared models.

\begin{tabular}{|c|c|c|c|c|c|c|c|c|}
\hline \multirow[b]{3}{*}{ Sequence Length } & \multicolumn{4}{|c|}{ MNIST cumulative sum } & \multicolumn{4}{|c|}{ MNIST cumulative product } \\
\hline & \multirow{2}{*}{$\begin{array}{c}\text { Acc. } \\
1\end{array}$} & \multicolumn{3}{|c|}{ MAE } & \multirow{2}{*}{$\begin{array}{c}\text { Acc. } \\
1\end{array}$} & \multicolumn{3}{|c|}{ log MAE } \\
\hline & & 5 & 10 & 100 & & 5 & 10 & 15 \\
\hline LSTM & $9.80 \%$ & 15.3008 & 44.3082 & 449.8304 & $9.80 \%$ & 11.1037 & 19.5594 & 21.6346 \\
\hline RNN-Relu & $10.32 \%$ & 12.3664 & 41.4368 & 446.9737 & $9.80 \%$ & 10.7635 & 19.8029 & 21.8928 \\
\hline DeepProblog & \multicolumn{4}{|c|}{ Training timeout (72 hours) } & $93.64 \%$ & \multicolumn{3}{|c|}{ Test timeout (72 hours) } \\
\hline LSTM-NAC & $7.02 \%$ & 6.0531 & 29.8749 & 435.4106 & $0.00 \%$ & 9.6164 & 20.9943 & 17.9787 \\
\hline LSTM-NAC $_{10 k}$ & $8.85 \%$ & 1.9013 & 21.4870 & 424.2194 & $10.50 \%$ & 9.3785 & 20.8712 & 17.2158 \\
\hline LSTM-NALU & $0.00 \%$ & 6.2233 & 32.7772 & 438.3457 & $0.00 \%$ & 9.6154 & 20.9961 & 17.9487 \\
\hline LSTM-NALU $_{10 k}$ & $0.00 \%$ & 6.1041 & 31.2402 & 436.8040 & $0.00 \%$ & 8.9741 & 20.9966 & 18.0257 \\
\hline Meta $a_{A b d}$ & $95.27 \%$ & 0.5100 & 1.2994 & 6.5867 & $\mathbf{9 7 . 7 3 \%}$ & 0.3340 & 0.4951 & $\mathbf{2 . 3 7 3 5}$ \\
\hline LSTM-NAC $_{1}$ & $49.83 \%$ & 0.8737 & 21.1724 & 426.0690 & $0.00 \%$ & 6.0190 & 13.4729 & 17.9787 \\
\hline LSTM-NALU $_{1 \text {-shot CNN }}$ & $0.00 \%$ & 6.0070 & 30.2110 & 435.7494 & $0.00 \%$ & 9.6176 & 20.9298 & 18.1792 \\
\hline Meta $_{A b d+1 \text {-shot CNN }}$ & $98.11 \%$ & 0.2610 & 0.6813 & 4.7090 & $97.94 \%$ & 0.3492 & 0.4920 & 2.4521 \\
\hline
\end{tabular}

Table 2: Accuracy on the MNIST cumulative sum/product tasks.

Cumulative Sum:

$f(A, B):-\operatorname{add}(A, C), f(C, B)$.

$f(A, B):-e q(A, B)$.

Cumulative Product:

$f(A, B):-\operatorname{mult}(A, C), f(C, B)$.

$f(A, B):-e q(A, B)$.

Bogosort:

$f(A, B):-$ permute $(A, B, C), s(C)$.

$\mathrm{s}(A):-\mathrm{s}_{-} 1(\mathrm{~A}, \mathrm{~B}), \mathrm{s}(\mathrm{B})$.

$s(A):-\operatorname{tail}(A, B), \operatorname{empty}(B)$.

$\mathrm{s}_{-} 1(A, B):-n n \_p r e d(A), \operatorname{tail}(A, B)$.

(a) Learned programs

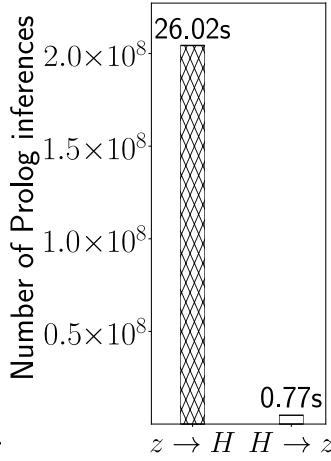

(b) Abduction time.
Figure 3: Learned programs and the time efficiency of $M e t a_{A b d}$.

The EM-based learning of $M e t a_{A b d}$ may be trapped in local optima, which happens more frequently in cumulative sum than produce since its distribution $P(H, z \mid B, x, y, \theta)$ is much denser. Therefore, we also carry out experiments with one-shot pre-trained convnets, which are trained by randomly sampling one example in each class from MNIST data. Although the pre-trained convnet is weak at start (Acc. $20 \% \sim 35 \%$ ), it provides a good initialisation and significantly improves the learning performance.

Fig. 3b compares the time efficiency between ILP's induction and $M e t a_{A b d}$ 's abduction-induction in one EM iteration of learning cumulative sum. " $z \rightarrow H$ " means first sampling $z$ and then inducing $H$ with ILP; " $H \rightarrow z$ " means first sampling an abductive hypothesis $H$ and then using $H$ to abduce $z$. The $\mathrm{x}$-axis denotes the average number of Prolog inferences, the number at the end of each bar is the average inference time in seconds. Evidently, the abduction leads to a substantial improvement in the number of Prolog inferences and significantly the complexity of searching pseudo-labels.

\subsection{Bogosort From Images}

Materials. We follow the settings in [Grover et al., 2019]. The input of this task is a sequence of randomly chosen MNIST images of distinct numbers; the output is the correct ranking (from large to small) of the digits. For example, when $x=\mid \boldsymbol{5}, \boldsymbol{4}, \mathbf{4}, \mathbf{3}, \mathbf{8}$ then the output should be $y=[3,1,4,5,2]$ because the ground-truth labels $z^{*}=$ $[5,9,4,3,8]$. The training dataset contains 3000 training/test and 1000 validation examples. The training examples are sequences of length 5 , and we test the learned models on image sequences with lengths 3,5 and 7 .

Methods. We compare Meta $a_{A b d}$ to an end-to-end model NeuralSort [Grover et al., 2019] and a state-of-the-art NeSy approach Neural Logical Machines (NLM) [Dong et al., $2019]^{5}$. All experiments are repeated five times.

NeuralSort can be regarded as a differentiable approximation to bogosort (permutation sort). Given an input list of scalars, it generates a stochastic permutation matrix by applying the pre-defined deterministic or stochastic sort operator on the inputs. NLM can learn sorting through reinforcement learning in a domain whose states are described by vectors of relational features (groundings of dyadic predicates" $>$ ", "==", " $<$ ") and action "swap". However, the original NLM only takes symbolic inputs ${ }^{6}$, which provides a noisy-free relational features vector. In our experiments, we attach NLM with the same convnet as other methods to process images.

\footnotetext{
${ }^{5}$ We use NeuralSort at https://github.com/ermongroup/neuralsort; NLM at https:/github.com/google/neural-logic-machines.

${ }^{6} \mathrm{Please}$ see https://github.com/google/neural-logic-machines /blob/master/scripts/graph/learn_policy.py
} 


\begin{tabular}{|c|c|c|c|}
\hline Sequence Length & 3 & 5 & 7 \\
\hline Neural Logical Machine (NLM) & $17.97 \%(34.38 \%)$ & $1.03 \%(20.27 \%)$ & $0.01 \%(14.90 \%)$ \\
\hline Deterministic NeuralSort & $95.49 \%(96.82 \%)$ & $88.26 \%(94.32 \%)$ & $80.51 \%(92.38 \%)$ \\
\hline Stochastic NeuralSort & $95.37 \%(96.74 \%)$ & $87.46 \%(94.03 \%)$ & $78.50 \%(91.85 \%)$ \\
\hline$M e t a_{A b d}$ & $96.33 \%(97.22 \%)$ & $91.75 \%(95.24 \%)$ & $87.42 \%(93.58 \%)$ \\
\hline
\end{tabular}

Table 3: Accuracy of MNIST sort. First value is the rate of correct permutations; second value is the rate of correct individual element ranks.

We also compared to DeepProblog with the ground-truth program of sorting in this task, but it does not terminate when the neural predicate "swap_net"7 is implemented to take noisy image inputs by the aforementioned convnet. Therefore, we do not display its performance in this task.

For $M e t a_{A b d}$, it is easy to include stronger background knowledge for learning more efficient sorting algorithms [Cropper and Muggleton, 2019]. But in order to make a fair comparison to NeuralSort, we adapt the same background knowledge to logic program and let $M e t a_{A b d}$ learn bogosort. The knowledge of permutation in Meta $a_{A b d}$ is implemented with Prolog's built-in predicate permutation. Meanwhile, instead of providing the information about sorting as prior knowledge like the NeuralSort, we try to learn the concept of "sorted" (represented by a monadic predicate s) from data as a sub-task, whose training set is the subset of the sorted examples within the training dataset $(<20$ examples). The two tasks are trained sequentially as a curriculum. $M e t a_{A b d}$ learns the sub-task in the first five epochs and then re-uses the learned models to learn bogosort.

$M e t a_{A b d}$ uses an MLP attached to the same untrained convnet as other models to produce dyadic probabilistic facts nn_pred( $\left.\left[\mathbf{7}, \mathbf{G}_{-}\right]\right)$, which learns if the first two items in the image sequence satisfy a dyadic relation. Unlike NLM, the background knowledge of $M e t a_{A b d}$ is agnostic to ordering, i.e., the dyadic nn_pred is not provided with supervision on whether it should learn "greater than" or "less than", so nn_pred only learns an unknown dyadic partial order among MNIST images. As we can see, the background knowledge used by $M e t a_{A b d}$ is much weaker than the others.

Results. Tab. 3 shows the average accuracy of the compared methods in the sorting tasks; Fig. 3a shows the learned programs by $M e t a_{A b d}$. The performance is measured by the average proportion of correct permutations and individual permutations following [Grover et al., 2019]. Although using weaker background knowledge, $M e t a_{A b d}$ has a significantly better performance than NeuralSort. Due to the high samplecomplexity of reinforcement learning, NLM failed to learn any valid perceptual model and sorting algorithm (success trajectory rate $0.0 \%$ during training).

The learned program of $s$ and the dyadic neural net nn_pred are both successfully re-used in the sorting task, where the learned program of $\mathrm{s}$ is consulted as interpreted background knowledge [Cropper et al., 2020], and the neural network that generates probabilistic facts of nn_pred is directly re-used and continuously trained during the learning of

\footnotetext{
${ }^{7}$ Please see https://bitbucket.org/problog/deepproblog/src/master /examples/NIPS/Forth/Sort/quicksort.pl
}

sorting. This experiment also demonstrates $M e t a_{A b d}$ 's ability of learning recursive logic programs and predicate inven-

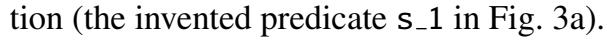

\section{Conclusion}

In this paper, we present the Abductive Meta-Interpretive Learning $\left(M e t a_{A b d}\right)$ approach that can simultaneously train neural networks and learn recursive first-order logic theories with predicate invention. By combining ILP with neural networks, $M e t a_{A b d}$ can learn human-interpretable logic programs directly from raw-data, and the learned neural models and logic theories can be directly re-used in subsequent learning tasks. Meta $a_{A b d}$ adopts a general framework for combining perception with logical induction and abduction. The perception model extracts probabilistic facts from sub-symbolic data; the logical induction searches for first-order abductive theories in a relatively small hypothesis space; the logical abduction uses the abductive theory to prune the vast search space of the truth values of the probabilistic facts. The three parts are optimised together in a probabilistic model.

In future work, we would like to apply $M e t a_{A b d}$ in real tasks such as computational science discovery, which is a typical abductive process that involve both symbolic domain knowledge and continuous/noisy raw data. Since $M e t a_{A b d}$ uses pure logical inference for reasoning, it is possible to leverage more advanced symbolic inference/optimisation techniques like Satisfiability Modulo Theories (SMT) [Barrett and Tinelli, 2018] and Answer Set Programming (ASP) [Lifschitz, 2019] to reason more efficiently.

\section{Acknowledgements}

The first author acknowledges support from the UK's EPSRC Robot Synthetic Biologist, grant EP/R034915/1, for financial support. The second author acknowledges support from the UK's EPSRC Human-Like Computing Network, grant EP/R022291/1, for which he acts as director. The authors thank Céline Hocquette, Stassa Patsantzis and Ai Lun for their careful proofreading and helpful comments.

\section{References}

[Barrett and Tinelli, 2018] Clark W. Barrett and Cesare Tinelli. Satisfiability modulo theories. In Handbook of Model Checking, pages 305-343. Springer, 2018.

[Bengio, 2017] Yoshua Bengio. The consciousness prior. CoRR, abs/1709.08568, 2017.

[Cohen et al., 2020] William W. Cohen, Fan Yang, and Kathryn Mazaitis. Tensorlog: A probabilistic database implemented using deep-learning infrastructure. Journal of Artificial Intelligence Research, 67:285-325, 2020. 
[Cropper and Muggleton, 2019] Andrew Cropper and Stephen H. Muggleton. Learning efficient logic programs. Maching Learning, 108(7):1063-1083, 2019.

[Cropper et al., 2020] Andrew Cropper, Rolf Morel, and Stephen Muggleton. Learning higher-order logic programs. Maching Learning, 109(7):1289-1322, 2020.

[Dai and Zhou, 2017] Wang-Zhou Dai and Zhi-Hua Zhou. Combining logical abduction and statistical induction: Discovering written primitives with human knowledge. In Proceedings of the 31st AAAI Conference on Artificial Intelligence, pages 43924398, San Francisco, CA, 2017.

[Dai et al., 2019] Wang-Zhou Dai, Qiu-Ling Xu, Yang Yu, and ZhiHua Zhou. Bridging machine learning and logical reasoning by abductive learning. In Advances in Neural Information Processing Systems 32, pages 2811-2822. Curran Associates, Inc., 2019.

[De Raedt et al., 2020] Luc De Raedt, Sebastijan Dumančić, Robin Manhaeve, and Giuseppe Marra. From statistical relational to neuro-symbolic artificial intelligence. In Christian Bessiere, editor, Proceedings of the 29th International Joint Conference on Artificial Intelligence, pages 4943-4950. IJCAI, 72020.

[Dhingra et al., 2020] Bhuwan Dhingra, Manzil Zaheer, Vidhisha Balachandran, Graham Neubig, Ruslan Salakhutdinov, and William W. Cohen. Differentiable reasoning over a virtual knowledge base. In International Conference on Learning Representations, Addis Ababa, Ethiopia, 2020. OpenReview.

[Donadello et al., 2017] Ivan Donadello, Luciano Serafini, and Artur S. d'Avila Garcez. Logic tensor networks for semantic image interpretation. In Proceedings of the 26th International Joint Conference on Artificial Intelligence, pages 1596-1602, Melbourne, Australia, 2017. IJCAI.

[Dong et al., 2019] Honghua Dong, Jiayuan Mao, Tian Lin, Chong Wang, Lihong Li, and Denny Zhou. Neural logic machines. In International Conference on Learning Representations, New Orleans, LA, 2019. OpenReview.

[Evans and Grefenstette, 2018] Richard Evans and Edward Grefenstette. Learning explanatory rules from noisy data. Journal of Artificial Intelligence Research, 61:1-64, 2018.

[Evans et al., 2021] Richard Evans, Matko Bošnjak, Lars Buesing, Kevin Ellis, David Pfau, Pushmeet Kohli, and Marek J. Sergot. Making sense of raw input. Artificial Intelligence, 299:103521, 2021.

[Flach et al., 2000] Peter A. Flach, Antonis C. Kakas, and Antonis M. Hadjiantonis, editors. Abduction and Induction: Essays on Their Relation and Integration. Applied Logic Series. Springer Netherlands, 2000.

[Garcez et al., 2019] Artur S. d'Avila Garcez, Marco Gori, Luís C. Lamb, Luciano Serafini, Michael Spranger, and Son N. Tran. Neural-symbolic computing: An effective methodology for principled integration of machine learning and reasoning. IfCoLog Journal of Logics and their Applications, 6(4):611-632, 2019.

[Gaunt et al., 2017] Alexander L. Gaunt, Marc Brockschmidt, Nate Kushman, and Daniel Tarlow. Differentiable programs with neural libraries. In Proceedings of the 34th International Conference on Machine Learning, volume 70, pages 1213-1222, Sydney, Australia, 2017. PMLR.

[Glasmachers, 2017] Tobias Glasmachers. Limits of end-to-end learning. In Proceedings of The 9th Asian Conference on Machine Learning, volume 77, pages 17-32, Seoul, Korea, 2017. PMLR.
[Grover et al., 2019] Aditya Grover, Eric Wang, Aaron Zweig, and Stefano Ermon. Stochastic optimization of sorting networks via continuous relaxations. In International Conference on Learning Representations, New Orleans, LA, 2019. Openreview.

[Hocquette and Muggleton, 2018] Céline Hocquette and Stephen H. Muggleton. How much can experimental cost be reduced in active learning of agent strategies? In Proceedings of the 28th International Conference on Inductive Logic Programming, volume 11105, pages 38-53, Ferrara, Italy, 2018. Springer.

[Kahneman, 2011] Daniel Kahneman. Thinking, fast and slow. Farrar, Straus and Giroux, New York, 2011.

[Kakas et al., 1992] Antonis C. Kakas, Robert A. Kowalski, and Francesca Toni. Abductive logic programming. Journal of Logic Computation, 2(6):719-770, 1992.

[Li et al., 2020] Qing Li, Siyuan Huang, Yining Hong, Yixin Chen, Ying Nian Wu, and Song-Chun Zhu. Closed loop neuralsymbolic learning via integrating neural perception, grammar parsing, and symbolic reasoning. In Proceedings of the 37th International Conference on Machine Learning, volume 119, pages 5884-5894, Online, 2020. PMLR.

[Lifschitz, 2019] Vladimir Lifschitz. Answer Set Programming. Springer, 2019.

[Manhaeve et al., 2018] Robin Manhaeve, Sebastijan Dumancic, Angelika Kimmig, Thomas Demeester, and Luc De Raedt. Deepproblog: Neural probabilistic logic programming. In Advances in Neural Information Processing Systems 31, pages 3753-3763, Montréal, Canada, 2018. Curran Associates, Inc.

[Muggleton and de Raedt, 1994] Stephen H. Muggleton and Luc de Raedt. Inductive logic programming: Theory and methods. The Journal of Logic Programming, 19-20:629 - 679, 1994.

[Muggleton et al., 2013] Stephen H. Muggleton, Dianhuan Lin, Jianzhong Chen, and Alireza Tamaddoni-Nezhad. MetaBayes: Bayesian meta-interpretative learning using higher-order stochastic refinement. In Proceedings of the 23rd International Conference on Inductive Logic Programming, volume 8812, pages 1-17, Rio de Janeiro, Brazil, 2013. Springer.

[Muggleton et al., 2015] Stephen H. Muggleton, Dianhuan Lin, and Alireza Tamaddoni-Nezhad. Meta-interpretive learning of higher-order dyadic datalog: predicate invention revisited. $M a-$ chine Learning, 100(1):49-73, 2015.

[Trask et al., 2018] Andrew Trask, Felix Hill, Scott E Reed, Jack Rae, Chris Dyer, and Phil Blunsom. Neural arithmetic logic units. In Advances in Neural Information Processing Systems 31, pages 8035-8044. Curran Associates, Inc., 2018.

[Wang et al., 2019] Po-Wei Wang, Priya L. Donti, Bryan Wilder, and J. Zico Kolter. SATNet: Bridging deep learning and logical reasoning using a differentiable satisfiability solver. In Proceedings of the 36th International Conference on Machine Learning, pages 6545-6554, Long Beach, CA, 2019. PMLR.

[Zhou et al., 2018] Jie Zhou, Ganqu Cui, Zhengyan Zhang, Cheng Yang, Zhiyuan Liu, and Maosong Sun. Graph neural networks: A review of methods and applications. CoRR, abs/1812.08434, 2018.

[Zhou, 2019] Zhi-Hua Zhou. Abductive learning: towards bridging machine learning and logical reasoning. Science China Information Sciences, 62(7), 2019. 